AperTO - Archivio Istituzionale Open Access dell'Università di Torino

\title{
Leveraging Web 3D guidance in cultural heritage fruition
}

\section{This is the author's manuscript}

Original Citation:

Availability:

This version is available http://hdl.handle.net/2318/146513

since 2016-11-30T12:33:00Z

Publisher:

Acm

Published version:

DOI:10.1145/2499149.2499157

Terms of use:

Open Access

Anyone can freely access the full text of works made available as "Open Access". Works made available under a Creative Commons license can be used according to the terms and conditions of said license. Use of all other works requires consent of the right holder (author or publisher) if not exempted from copyright protection by the applicable law. 


\section{Leveraging Web 3D Guidance in cultural heritage fruition}

\author{
Rossana Damiano \\ Dipartimento di Informatica and CIRMA \\ Universita' di Torino - Italy \\ Vincenzo Lombardo \\ Dipartimento di Informatica and CIRMA \\ Universita' di Torino - Italy \\ Virtual Reality and Multimedia Park
}

\author{
Cristina Gena \\ Dipartimento di Informatica and CIRMA \\ Universita' di Torino - Italy \\ Fabrizio Nunnari* \\ Universita' di Torino - Italy \\ Virtual Reality and Multimedia Park
}

\begin{abstract}
This paper describes a 3D tour of exhibitions set up for the celebration of the 150 years of Italian unity. Through a specifically developed Web plugin, the users can navigate the reconstruction of the exhibitions and receive information about the exhibits. The 3D tour is embedded in a Web 3.0 portal (name omitted for blind review) designed for the promotion and dissemination of the exhibitions. The portal integrates the social and the Web 3D components in an immersive environment, where users can switch from the 3D visit to the standard hypertext-base visit or take advantage of recommendations and obtain information without abandoning the 3D environment. In this paper, we describe the design and technologies that characterize the $3 \mathrm{D}$ visit and its experimental evaluation, conducted on real users.
\end{abstract}

Keywords: Web 3D, Web 3.0, evaluation, learning enviornments

\section{Introduction}

The use of Web 3D in cultural heritage promotion allows the general public to live immersive experiences in virtual, reconstructed locations, like ancient towns and locations (see, e.g. [Calori et al. 2008; Cabral et al. 2007]), and to visit existent, but remotely located locations, such as world-wide cultural institutions (such as Google Art Project ${ }^{1}$ ). For preservation purposes, web 3D provides scholars and cultural heritage professionals with a way to consult and maintain visual repositories of real exhibits, with the possibility of visualizing, comparing and studying 3D digital equivalents of real artworks physically situated in different locations (see, e.g., [Rodriguez-Echavarria et al. 2009]).

Using the Web as a platform for 3D applications enables the integration of 3D tools into an environment users are familiar with, making Web 3D part of the Web 3.0 experience. Web 3.0 sites, in fact, exploit social and semantic technologies to provide innovative services, such as semantic search, personalization, etc., in conjunction with 3D data and augmented reality. These features are encompassed by the definition of Web 3.0 provided by W.L. Hosch as the "executable web". In the analogy to file system permissions, Web

*e-mail:nunnarif@vrmmp.it
1.0 was "read-only", Web 2.0 is "read-write", and Web 3.0 will be "read-write-execute" 2 .

However, the Web and 3D technologies are at odds for a number of factors. On the one side, the distribution of Web contents still suffers from bandwidth limitations, while the use of 3D poses high requirements on bandwidth and data transmission. On the other side, the navigation paradigm of standard Web clashes with the need for guidance that characterizes 3D applications, thoroughly discusses in [Jankowski 2011]. Notwithstanding these contradictions, the use of Web 3D is bound to become still more widespread with the increasing diffusion of mobile devices (such as smartphones and tablets), also given its ability to attract the attention of young people and video game users.

Given these considerations, an open research problem in Web 3D consists in finding the right balance between guidance and user initiative in Web 3D applications. In this paper, we describe the design and evaluation of a 3D tour of the exhibitions set up for the celebration of the 150 years of Italian unity. The application we propose trades off immersiveness and guidance by anchoring the 3D contents to the information structure of the web site, where they are accompanied by social and semantic tools. After describing the 3D tour and the technologies that support it, we illustrate the evaluation we conducted of the 3D tour - where we compare our application, and its crucial points, against the baseline of a commercial project, namely Google Art Project — and discuss the results.

Embedded in a Web 3.0 portal designed for the publicity and dissemination of the exhibitions that describe the birth of Italy as a national state, the 3D visit allow the users to navigate the reconstruction of the exhibitions and receive information about the exhibits. Targeted at schools, the web portal encourages the active participation of the users (students, teachers, registered users) in different ways: by tagging and commenting, with their own words, the exhibition contents, by expressing their likes and dislikes through votes, by creating new contents. Following the paradigm of the Web 3.0, known as "the executable web", the portal integrates the social and the Web 3D components in an immersive environment, where users can switch from the 3D visit to the standard hypertext-base visit and take advantage of recommendations and obtain information without abandoning the 3D environment.

This paper is structured as follows. Section 2 provides a general description of the project. The 3D visit is described in Section 3 with its implementation and design. Google Art Project is described in Section 4. Section 5 reports the evaluation, their results, and comparison, while Section 6 presents related work. Conclusions end the paper.

\footnotetext{
${ }^{2} \mathrm{http}: / /$ www.britannica.com/blogs/2007/07/web-30-the-dreamer-of-thevine/ 


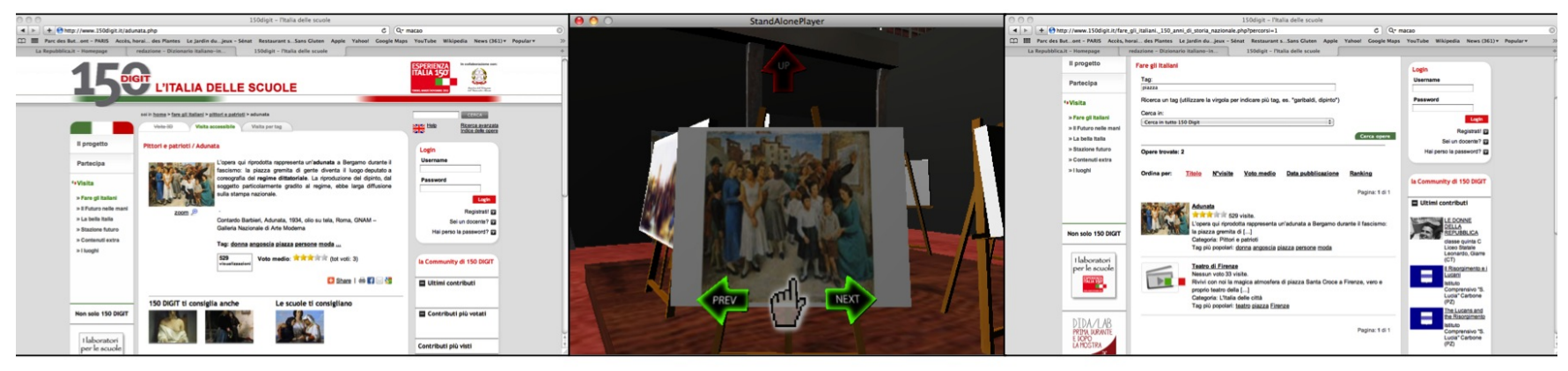

Figure 1: The visit modalities in the Web 3.0 portal. Left, standard hypertext; center, 3D visit; right, tag-based.

\section{Portal Description}

The goal of the Web 3.0 portal is to implement an open environment where students can visit the exhibitions online and access a wide repository of media items related with the subject of the Unity of Italy, a process achieved 150 years ago, in 1861 . The repository contains both institutional contents, taken from the exhibitions, and user-generated contents. Contents can be tagged and annotated by the users, thus inducing new connections over the exhibits, and can be accessed via tags. User preferences and tags are used to generate recommendations and promote the exploration of contents in a "bottom-up" perspective, in parallel with the institutional classification of the exhibits enforced by the architecture of the site.

The site relies on the metaphor of the 'visit' to structure the information. The user can visit the four main exhibitions though a 3D tour, a standard hypertext-based visit, or by searching tags. Moreover, he/she can switch from one modality to another, and remain in the same (virtual) location.

The project encompasses three user profiles: the editor, who is in charge of editing and publishing the institutional contents provided by the curators, and validating the contents uploaded by the students; the student/teacher, who can visit the exhibitions, add tags and comments to the exhibits, vote them, and upload new items; the registered user, who can visit the exhibitions, vote and tag the exhibits and create her/his own playlist in a private area. Given these profiles, the portal has three main functions: content classification and publication; content editing; navigation and visualization.

The portal relies on a semantic layer to to improve content description and recommendation, and features a plugin, tested on major browser, for navigating the exhibitions in a 3D environment, with the aim of making the access to the exhibits more compelling and less mediated by text. This approach borrows from entertainment (and videogames in particular) to offer students with a modality of access they are familiar with. The standard visit of the exhibitions, structured along a hierarchical classification of the exhibits (including user-generated contents), is accompanied by two alternative modalities of visit: the $3 \mathrm{D}$ visit of the exhibitions and the tag-based visit (see Figure 1).

The Web 3.0 portal has been developed by a multi-disciplinary team, involving AI, computer graphics, interaction design and media experts, and with the participation of the target users along all the phases of the project, from design to prototyping, according to an iterative design methodology (see for details [Damiano et al. 2011]). The resulting portal integrates different components in a seamless interface that overcomes the challenges posed by the software integration issues and the content production process.

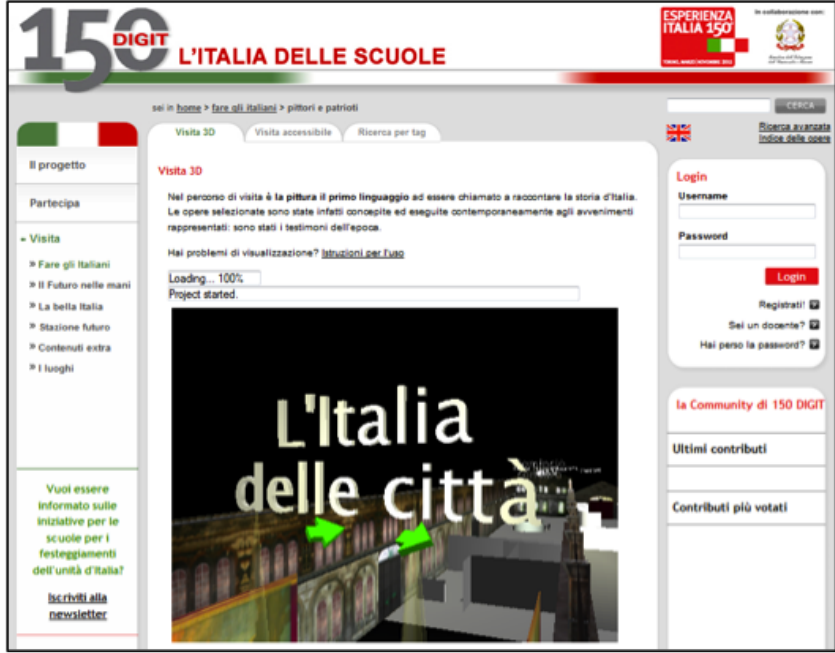

Figure 2: A snapshot of the 3D visit of the exhibition "Fare gli Italiani”.

\section{3D Visit}

The 3D visit of the Web 3.0 portal was conceived as a constrained spatial navigation that allows the access to a selection of the exhibition items [Burigat and Chittaro 2007]. The visit is partially constrained to some fixed positions, in a sequential order, where the visitor is "transported" through a stepwise flight simulation.

The curators of the exhibitions have classified the items according to a number of categories, that are listed in the accessible visit. A Java applet provides access to a 3D model of the exhibition space, where models of the items are exposed according to the layout of the real exhibition.

\subsection{D Visit: Interaction Design}

An initial camera motion provides an overview of the exhibition and gets the user to the first group (category) of exhibits. Each group is represented by a 3D text with the label of the group; this label hovers over the actual position of the items (see Figure 2).

In front of each label, there are three signs (see Fig. 3):

1. an arrow oriented to the NEXT group (in case of the last group, a special arrow marked START points to the first group); 


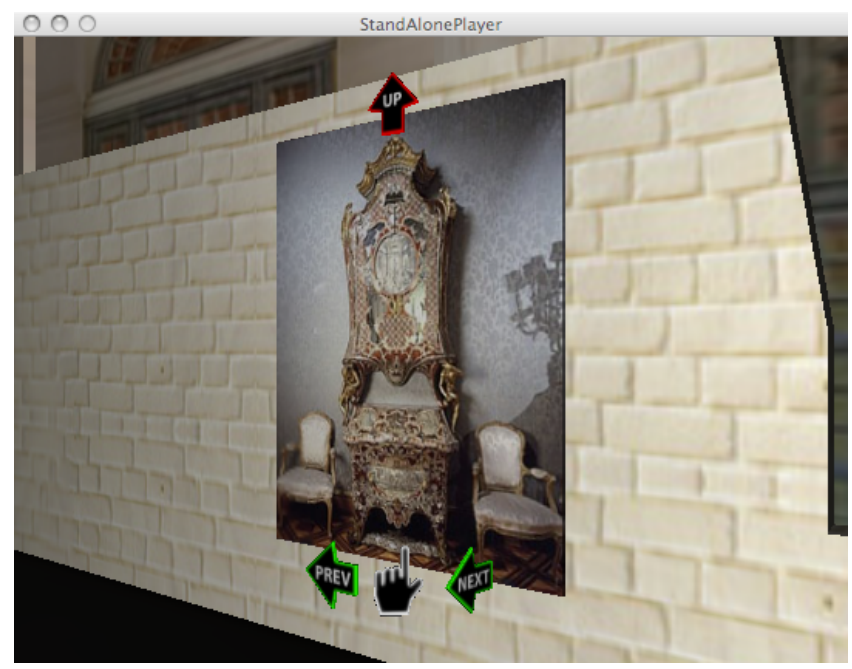

Figure 3: A screenshot of the $3 D$ visit of the exhibitions. Notice the navigation controls (arrows and hand-shaped control).

2. an arrow oriented to the PREVious group (in the case of the first group, a special arrow marked END points to the last group);

3. a hand-shaped icon that points to the label itself.

By clicking the arrows, the visitor is transported to another group label; by clicking the hand, the visitor descends to the items, and is positioned in front of the first item. Again, the system of the three signs allows the visitor to get to the next item of the group (NEXT arrow), the previous item of the group (PREV arrow), the information about the item itself (hand-shape icon). These signs allow a kind of direct guidance [Brusilovsky 2001], an adaptive navigation technique that proposes to users a strict linear order through the navigation space.

Each click causes a transportation to another fixed location and a callback to the web server to load a page related to the destination of the transportation. So, the web site is constantly updated with information aligned with the $3 \mathrm{D}$ visit. When the user gets to a single item, the callback triggers the loading of the information related to the item itself and the possibility to launch the player of the item, namely audio or video streaming, PDF opening, image display.

Finally, some keys have been programmed to control the camera orientation and motion. These keys are the standard PC keyboard associations for videogame interactions (W going ahead, S going back, ); so, expert visitors can take advantage of such recreational possibilities.

\subsection{D Visit: Development and Editing}

MESH (Mise-en-scène Helper) is a software platform for authoring environments in computer graphics. ${ }^{3}$ The MESH architecture relies upon a number of opensource libraries that account for the major modules of real-time computer graphics, from the rendering engine to the simulation of rigid body physics. The architecture allows for the import of scenes and characters from a number of authoring tools, including Google SketchUp. The MESH software architecture consists in a core platform, called Enthusiasm ${ }^{4}$, that includes the high-level graphic framework Tarta4D, the Sound Man-

\footnotetext{
${ }^{3}$ http://www.edu.vrmmp.it/mesh/

${ }^{4}$ enthusiasm.sourceforge.net
}

ager, and the graphic interface Director Studio, with the associates Control Score Player.

The core of the scene rendering is the Tarta4D Framework, a rendering library and 3D engine that offers high-level functionalities: Graphics (import of 3D objects authored with the most popular 3D authoring tools, realtime 3D rendering, 3D objects animation, automated animation blending); audio (spatialized 3D audio, playback of pre-loaded samples or in streaming mode); physics; multiplatform support (Windows, Linux, MacOS X); simplified scene management; multi-thread support, $\mathrm{C}++$ and Java APIs and Effortless integration in Java AWT/Swing interfaces.

The Tarta4D API (Application Programming Interface) exposes a simplified scenetree approach. A scene is defined as a tree of objects, cameras and lights. All the technical details about the reuse of resources (materials, geometries, skeletons, and the like) is completely automatized and hidden to the end user. A 3D Object in Tarta4D is a complex entity associated to a visual aspect, able to emit sounds, and responsive to gravity and collisions. A Scene is a container of objects characterized by light condition (sun light and ambient light) and a Root Object. Starting from the Root, the Objects are organized as a hierarchical tree. Scenes can be saved to and loaded from files, in an XML format.

Thanks to its Java wrapper, the Tarta4D library can be easily used to deploy an applet running into a web page. This is done by tacking advantage of an Ogre3D feature which allows the programmer to specify the surface that can be used for the 3D rendering. A rendering surface can be a window, or a portion of it, that has been already allocated by the operating system. This binding, which has been implemented on the Window and MacOS X platforms, allowed to easily create an applet by simply instantiating a Tarta4DCanvas inside our player (EnthusiasmStandalonePlayer), which extends the Applet class. The deployment of the applet has been done using the Java Web Start technology ${ }^{5}$, which provides all the facilities to transfer the required native libraries to the client machine. With respect to the development of ad-hoc plugins for specific browsers, our approach has the great advantage that the applet works on any browser supporting Java applets. The applet has been successfully tested on Explorer, Firefox, Opera and Chrome on WindowsXP, Vista and 7, and on Safari, Firefox and Chrome on the Mac OS X platform.

From an authoring point of view the interaction between the applet and its surrounding web page is performed through javascript callbacks. Each time "a callback" is raised by the 3D content (for example, after the user clicked on an object), a corresponding "callback" javascript is called, carrying a generic string parameter. This allows the web page developers to execute the appropriate action, such as updating page sections or showing a pop-up, according to the string parameter content. The Director Studio (see (see Fig. 4) is a set of windows that allows the author to control the initial layout and the dynamic behavior of all the elements of a scene, namely object instances, cameras, lights, animated characters, paths for the displacement of cameras and characters. As for the majority of authoring tools, the elements are arranged onto a hierarchy, with the possibility of represent the composition of complex objects in an analytic way. A MESH project consists of many scenes, each associated with a layout and a number of control scores. The graphic interface is organized in three panes: assets, scene layout, and control scores. The asset pane allows for the selection of a scene of the project and the setting of an environmental/sun light.

\footnotetext{
${ }^{5}$ http://download.oracle.com/javase/6/docs/technotes/guides/javaws/
} 


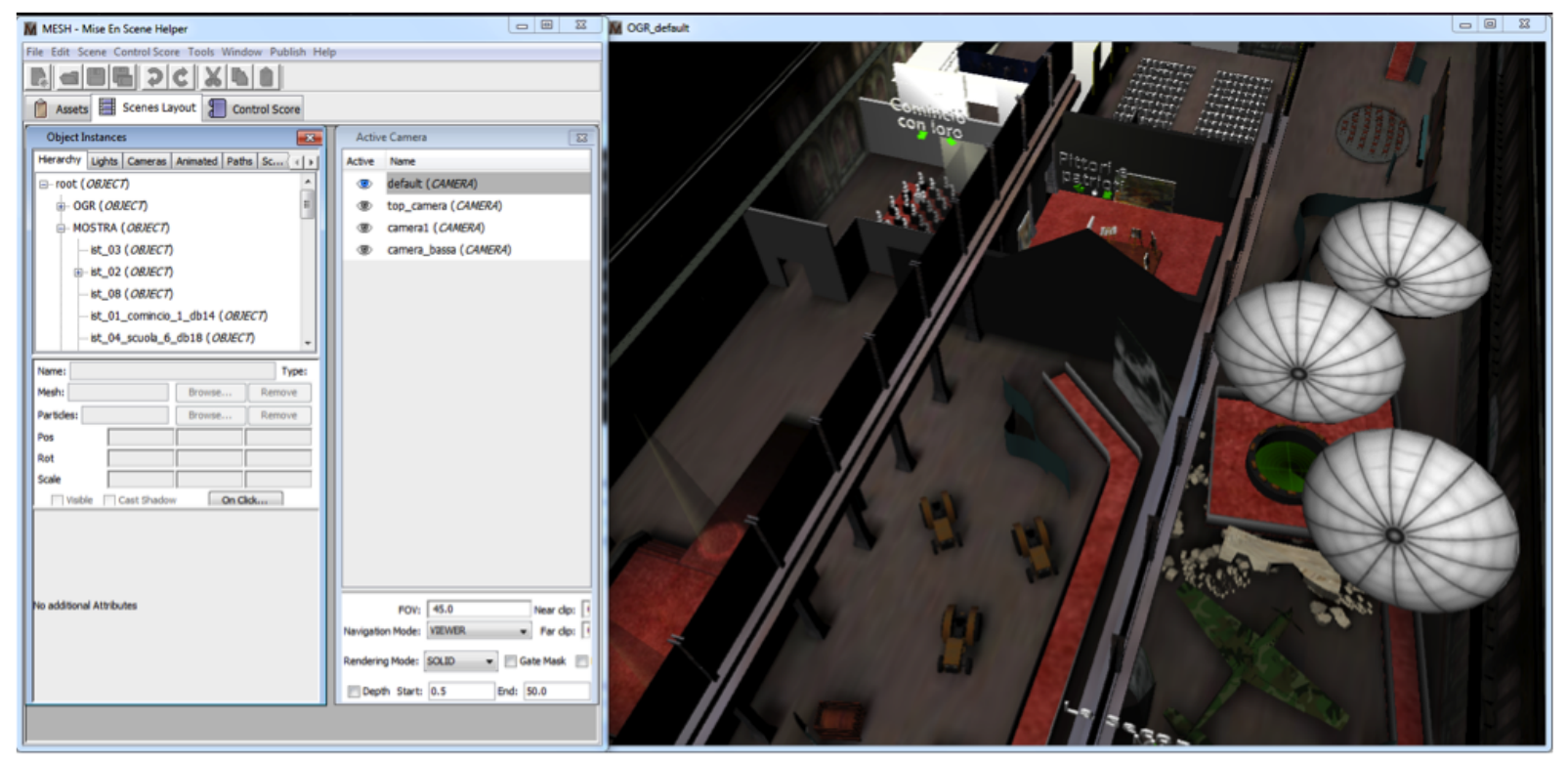

Figure 4: The making of the 3D visit with the Enthusiasm tool (Director Studio).

\section{The Google Art Project}

In order to have a benchmark with a commercial high-level tool, we have compared our application against the baseline of a commercial project, namely Google Art Project.

Since February 2011, Google, in collaboration with most important museums of the world, lets web users freely navigate through museums' artworks in its Google Art Project. Thanks to the technology of Google Street View the user can observe and scatter over a thousand paintings in the seventeen museums that have initially joined the initiative and have offered their advice for provide the best possible works. Google Art offers real virtual tours to appreciate the works thanks to the high resolution images. Among the participating museums there are the MoMA and The Metropolitan Museum of Art in New York, the Tate Gallery and the National Gallery in London, the Van Gogh Museum in Amsterdam, the Palace of Versailles and the Italian Uffizi Gallery.

The technology behind Google Art Project is the same as Google Street View: clicking on the screen the user moves through the galleries of the chosen museum and can see details invisible to the naked eye thanks to a set of artwork's images photographed with a significant level of resolution.

The navigation system at 360 degrees - this feature is made easier by the presence of the Google Earth rotating widget on the topleft corner of the interface - and a powerful zoom allows detailed observation of artworks (Artwork View mode) enabling the user to see even the smallest details, usually not visible to the naked eye. As for the navigation in Google Street View, the user may use system arrows to move with the mouse, but she can also use keyboard arrows (see Figure 5).

For each virtual museum is available an interactive map that allows users to move through museum rooms or read in detail the history of the museum, of the chosen artist, and of the artwork itself. For every artwork the user can easily find other works on the same author in the museum or her works in other museums. All these features are accessible from the right side panel.

Much attention was also placed in the social features of virtual mu- seums, such as for related video on Youtube. Moreover, every virtual visitor will be able to also create a personal gallery of artworks, through the function "Create an Artwork Collection", which can be commented and shared with friends and social networks.

In April 2012 Google launched an expanded version of the project that cover now 46 museums, with more than 30,000 objects (including not just paintings, but also sculpture, street art, and photographs) available to view in high resolution. The new Google Art project also includes completely new navigation tools and a new interface. Since our evaluation has been carried out in March 2012, subjects experienced the old version of Google Art Project. We will describe in section 5.2.3 the new tools and the new interface version. We will compare the new solutions provided by Google with the issues raised by the evaluation of the previous release of the system.

\section{Testing usability in the Web 3.0 portal and Google Art Project}

Google Art Project is an important benchmark for our study not only because is a high-level commercial tool, but also because shares a guidance/ user initiative approach similar to the one proposed in the 3D tour of the Web 3.0 portal. There are several similarities, but also differences between the two different systems. The 3D tour into the Web 3.0 portal and Google Art visit have the following similarities:

- user starts the 3D navigation by first selecting a museum;

- they both present 3D virtual environments to be either freely visited or visited by following a guidance given by system arrows;

- they both let the user to get more information about an artwork by clicking on it (and the more information are presented outside the virtual environment);

- they both use 3D widgets to let the user navigate into the environment (e.g., the arrows) and to accomplish some task (getting more information by clicking on a icon); 

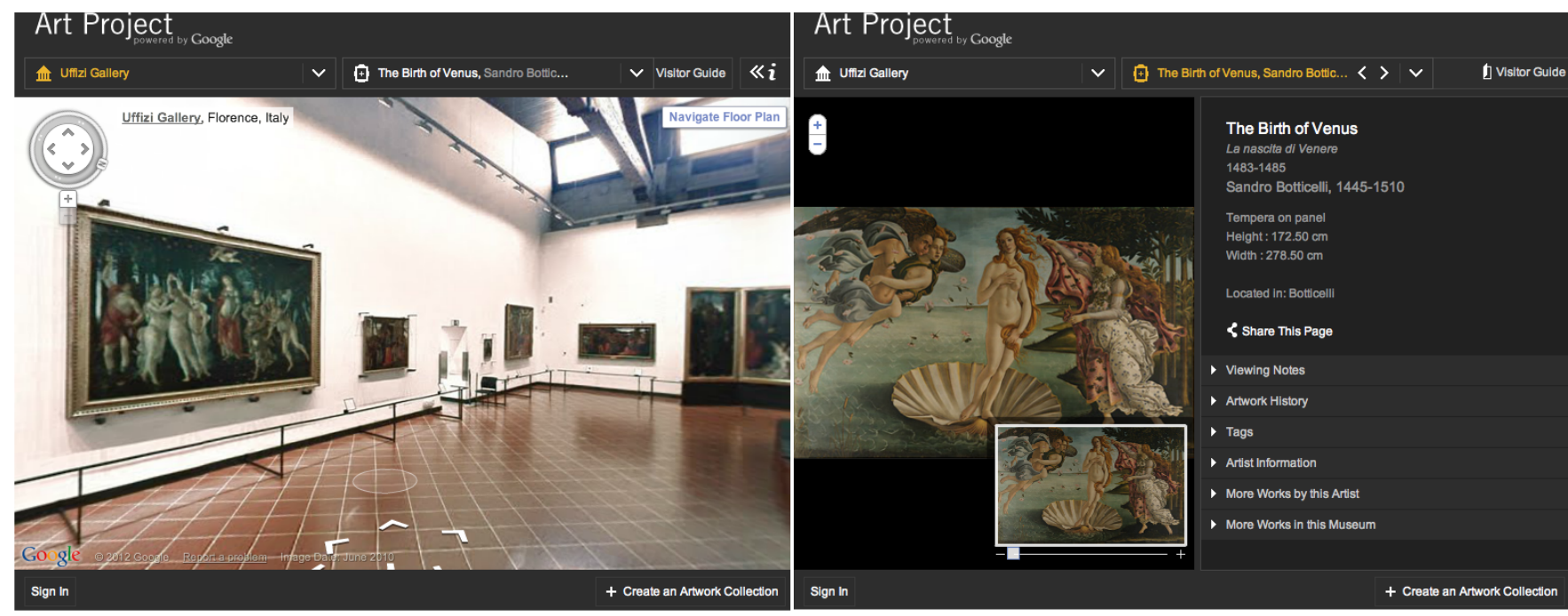

Figure 5: Google Art: Street view navigation interface (left side) and Artwork View mode (right side)

- they both offer Web 2.0 features (e.g., tagging, sharing, etc);

- they both offer alternative ways of searching and browsing between collections (by entering the artwork's title, the name of the author, by looking for museum spatial information, etc.);

and differences

- the 3D visit in the Web 3.0 portal is a virtual visit, completely reconstructed, while the Google Art visit aims at being very close to the real visit in the partner museum. In order to accomplish this goal, Google uses a special Street View trolley that took 360 degree images of the interior of the galleries;

- the Web 3.0 portal arrows impose a constrained visit into the environment, while the Google Art arrows specify a trajectory movement, similar to a cinematographic camera movement (see below);

- Google Art visit is very similar to experience a user may already have had (Google Street View), while the 3D tour is a completely new experience.

The tasks a user can perform in the 3D visit of the Web 3.0 portal can be classified according to the taxonomy of tasks (tasksonomy) in Web 3D by Jankowsky [Jankowski 2011]. Jankowsky distinguishes five subclasses of 3D user tasks: Navigation, Wayfinding, Selection, Manipulation and System Control. The user navigation in the 3D tour can be classified as Point of Interest (POI) Logarithmic Flight, a kind of a Targeted Movement. POI requires the user to simply choose a target "point of interest" on an object in the scene by using the mouse, and the virtual camera moves logarithmically towards this target, as the user does when selecting a group of artworks in the web 3.0 portal. Since the user is also free to move in $3 \mathrm{D}$ space using the keyboard arrows, the 3D user navigation can be classified into General Movement as well, a subtask that covers exploratory movement such as walking and examining. The Wayfinding task, which is related to how people build up an understanding (mental model) of a virtual environment, in the 3D visit 1 is accomplished by using a pre-determined path that is first of all proposed to the user as a constrained navigation.

Jankowsky's Navigation, Wayfinding, and Selection in the described 3D visit share some similarities with the first release of the Google Art. As for the navigation in Google Street View, the user may use system arrows to move following a Trajectory Movement, similar to a cinematographic camera movement, but she can also use keyboard arrows (Navigation - General Movement). When the user is interested on an artwork, she can click on the screen (on a widget similar in all the selectable artworks) and see details invisible to the naked eye thanks to a set of artwork's images photographed with a significant level of resolution. For each virtual museum is available an interactive map (Wayfinding - Use of Maps) that allows users to move through museum rooms or read in detail the history of the museum, of the chosen artist, and of the artwork itself. For every artwork the user can easily find other works on the same author in the museum or her works in other museums.

\subsection{Usability testing in the Web 3.0 portal}

The current section describes a usability test we run at the end of the development of the 3D tour of the Web 3.o portal. The goal of the test was to assess the effectiveness of the interaction design strategy in general (guidance vs. user initiative), and specific interaction aspect in particular, such as the usefulness of the arrow and their specific features (e.g. orientation, labels, etc.), the camera movements, the feeling of disorientation, the labeling, etc.

\subsubsection{Method}

Design. We run a usability test covering all the four interactive exhibitions.

Participants. There were 5 participants performing all the tasks for each exhibition (see Table 1 for details). There were 4 women, and 1 man. The participant were researchers in computer science and ranged in age from 30 to 37 . All participant daily use computer and web, at home and at work. Only one is a frequent video games user, and 2 of them had some experience in web 3D. Participants were a convenience sample recruited personally by the experimenters from the Department of Computer Science, University of. Participants started the test from a randomly chosen exhibition.

Apparatus and Materials. A computer (Apple MacBook Pro) was used for task execution. Users browsed the virtual museum using the Mac stand alone player. The performances were recorded by means both of a screen capture software and of a video camera. Users were given written instructions and had to fill in a pre-test and a post-test questionnaire. 


\begin{tabular}{|c||c|c|c|c|c|c|c|c|}
\hline Users & Sex & Age & Weekly Computer Usage & Internet Usage & Internet Usage & 3D Usage & Web 3D Usage & Visited Ex.? \\
\hline$\# 1$ & F & 31 & daily & more than 10 hours & work, fun & never & never & yes \\
\hline$\# 2$ & F & 37 & daily & more than 10 hours & work, fun, study & very often & sometimes & no \\
\hline$\# 3$ & F & 35 & daily & more than 10 hours & work, fun & never & never & yes \\
\hline$\# 4$ & M & 35 & daily & more than 10 hours & work, fun & sometimes & never & no \\
\hline$\# 5$ & F & 30 & daily & more than 10 hours & work, fun, study & sometimes & sometimes & no \\
\hline$\# 6$ & F & 23 & daily & 2-6 hours & work, fun, study & sometimes & sometimes & no \\
\hline
\end{tabular}

Table 1: Users in the first experiment (\#1 to \#5) and second experiment (\#1 to \#4 and \#6)

\begin{tabular}{|c||c|c|c|c|c|c|c|}
\hline 1(v. bad) to 5 (excellent) & $\mathbf{\# 1}$ & $\mathbf{\# 2}$ & $\mathbf{\# 3}$ & $\mathbf{\# 4}$ & $\mathbf{\# 5}$ & Av. & S.D. \\
\hline finding information & 2 & 2 & 2 & 4 & 5 & 3.00 & 1.41 \\
\hline virtual movements & 2 & 2 & 3 & 3 & 2 & 2.4 & 0.55 \\
\hline labeling comprehensibility & 5 & 5 & 4 & 3 & 4 & 4.2 & 0.84 \\
\hline contents completeness & 2 & 3 & 3 & 4 & 3 & 3.00 & 0.71 \\
\hline pleasantness of experience & 3 & 2 & 3 & 4 & 3 & 3.00 & 0.71 \\
\hline
\end{tabular}

Table 2: Quantitative results of the first experiment

Procedure. Participants took part in the experiment individually. They had to read written instructions in their own time. Opening instructions specified that the test was not aimed at assessing their abilities but at evaluating the 3D tour of the Web 3.0 portal. After the opening instructions participants filled in a pre-test questionnaire for collecting socio-demographic information and assessing their skills in using computer and their experience with video games and 3D web sites (see Table 1). The testing session began with a set of experimental tasks, different for each exhibition. Written instructions highlighted that if the user had any questions during the task execution she could ask, but only if she had no idea how to behave, since we were interested in seeing how users use the site when they are alone. The user could ask questions only if she intended to abandon the task performance.

The test presented a sets of tasks to execute during the $3 \mathrm{D}$ visit, in order to guide the user during the tour. Examples of tasks were: moving from a fixed location to another; visiting a group of items by clicking on the arrows; visiting another group of items by using the keyboard arrows; select an artwork the user likes and looks for more information; etc. When the path ended the user could switch to the next visit. The users were observed during the visits, while the testing monitor and the observer (silently and without affecting the respondent) have noted the user's behavior and the doubts, the critical points, the errors committed by the user, etc.

A the end of the test participants were required to complete a post hoc questionnaire about their experience (see Table 2). Participants used a 5-points scale to make their ratings, anchored at 1 (worst) and 5 (best). Finally the test monitor made a structured interview to each user investigating major problems, desired functionalities, problems with arrows and camera movements, etc.

\subsubsection{Results}

After an initial disorientation, 4 of 5 users moved with great ease thanks to the presence of arrows ("arrows are ugly but useful", user \#1 said). Guiding arrows were initially not very clear for users: it was not immediately clear that in a 3D environment you must first follow an imposed path rather than freely navigate and click on things. Arrows were particularly appreciated during the transportation between groups of artworks (arrows let simulate simulate a kind of flight between locations), unlike "start" and "end" arrows that have never been used ("I have not even noticed", user 4 said). When users were visiting a location containing a group of artworks, most of them preferred moving by means of keyboard standard associations and freely visiting the environment. A major problem emerged in this situation has been that artwork were not clickable for having more information, since the fixed "clickable hand" does not appear when user freely moves.

The different orientation degrees of the guiding arrows did not cause problems (a part from a user), unlike what was expected. Camera movement were not perceived too fast, and no one was bothered by the fact of passing through things. Sometimes users did not clearly understand which was the item in focus

Most of the users wished to have the possibility of quickly coming back to the start of the visit path, as well as having the possibility to always know where they are with respect to environment, where they have already been, and how long the visit is. 2 users suggested implementing a map overview - with "you are here" pointer, as in real museum - in order to avoid feeling lost in the virtual space. The two 3D experienced users also suggested to insert the name of the artworks also in the 3D environment, and to offer the "more information" fruition directly in the 3D (e.g. in the Second Life style) as well and avoiding to open a new browser window for that ("in order to have a more immersive experience", user \#2 said).

Final results of post hoc questionnaires (see Table 2) showed that i) looking for information was quite easy; ii) the movement in the hyperspace were not so easy as expected (probably due to the poor implementation of keyboard movements); iii) label's arrows were comprehensible; iv) information were exhaustive, and v) the experience was enjoyable.

Finally we detailed the answers of a specific question asked during the structured interview ("What kind of experience would compare this experience?YouTube - Google Street View - Google Art Project - videogames - Other"): for user \#1 3D the Web 3.0 portal is more immersive than Google Street View; for user \# 2 the experience is similar to Google Street View but also to Second Life; for user \#3 the Web 3.0 portal is similar to videogames; for user \#4 is similar to Google Street View and videogames, and opposite to Second Life since here the visit is structured; for user \#5 Google Street View and more in general to panoramic views.

\subsubsection{Discussion}

Test results have showed a quite good impact of the interaction design strategies on final users, however some change was required to enhance the user experience. After having analyzed the test results, and discussed them with the 3D team, we have decided to implement the following re-designs:

- feedback for the initial loading time during installation;

- artworks always clickable, so that user can get more information even when freely navigate;

- "Home" button always present;

- item in focus made clearer through the use of light. 
What in general seemed to emerge as requirement from this test is the user desire to freely move in the 3D space and to click on artworks whenever she wants to; the user's need of always knowing where she is and how to get back at the beginning of the visit (need of clear mechanisms of orientation in the 3-dimensional space); the user's need of textual information in the 3D visit as it happens in the real world.

\subsection{Testing usability in Google Art}

The current section describes a usability test of Google Art. The goal of the test was to assess the effectiveness of the interaction design strategy in general (guidance vs. user initiative), and specific interaction aspect in particular such as the navigation in Street View-based style, the arrow guidance strategies, the usefulness and the managing of the right-hand panel, the feeling of disorientation, etc.

\subsubsection{Method}

Design. We run a usability test covering four museum: Van Gogh Museum Amsterdam, Uffizi Gallery Florence, National Gallery London, Tate Britain London. The museum were chosen on the basis of the direct experience of the tester, who has visited these museum in the past. The tester experience was used used in order to better articulate the experimental tasks.

Participants. There were 5 participants performing all the tasks for each exhibition. There were 4 women, and 1 man (the same but one of the previous experiments). The new participant was a 23 years old female student attending a master in Multimedia and Communication Technologies. She daily uses the computer and spends 2-8 hours in Internet per week, and she had some experience in 3D web sites. Participants started the test from a randomly chosen exhibition.

Apparatus and Materials. A computer (Apple MacBook Pro) was used for task execution. The performances were recorded by means of a screen capture software. Users browsed virtual museum using the Safari web browser. Users were given written instructions and had to fill in a pre-test and a post-test questionnaire.

Procedure. Participants took part in the experiment individually. They had to read written instructions in their own time. Opening instructions specified that the test was not aimed at assessing their abilities but at evaluating the Google Art virtual visits. They were were alerted to the presence of the screen capture software and of the camera. They were asked to think aloud while performing the tasks. After the opening instructions participants filled in a pre-test questionnaire for collecting socio-demographic information and assessing their skills in using computer and their experience with video games and $3 \mathrm{~d}$ web sites (see Table 1). The testing session began with a set of experimental tasks, different for each exhibition. Written instructions highlighted that if the user had any questions during the task execution she could ask, but only if she had no idea how to behave, since we were interested in seeing how users use the site when they are alone. The user could ask questions only if she intended to abandon the task performance.

The test presented a sets of tasks to execute during the virtual visit, in order to guide the user during the tour. Examples of tasks were: moving from a room to another by means of either the floor plan map or the system/keyboard arrows; look for a specific artwork of the museum; select an artwork during the visit and and look for more information/tags; magnify a selected artwork; look for more information about a room; find all the artwork of a specific artist; etc.

\begin{tabular}{|c||c|c|c|c|c|c|c|}
\hline 1(v. bad) to 5 (excellent) & $\mathbf{\# 1}$ & $\mathbf{\# 2}$ & $\mathbf{\# 3}$ & $\mathbf{\# 4}$ & $\mathbf{\# 5}$ & Av. & S.D. \\
\hline finding information & 4 & 3 & 5 & 5 & 4 & 4.20 & 0.84 \\
\hline virtual movements & 3 & 2 & 3 & 3 & 4 & 3.00 & 0.71 \\
\hline labeling comprehensibility & 4 & 4 & 4 & 5 & 3 & 4.00 & 0.71 \\
\hline contents completeness & 5 & 1 & 5 & 5 & 4 & 4.00 & 1.73 \\
\hline pleasantness of experience & 5 & 2 & 4 & 4 & 4 & 3.80 & 1.10 \\
\hline
\end{tabular}

Table 3: Quantitative results of the second experiment

At the end of the test participants were required to complete a post hoc questionnaire about their experience (see Table 3). Participants used a 5-points scale to make their ratings, anchored at 1 (worst) and 5 (best). Finally the test monitor made a structured interview to each user investigating major problems, desired functionalities, problems with arrows and camera movements, etc.

\subsubsection{Results}

Almost all the user (4 out of 5) have experienced initial difficulties in finding the information contained in the sliding right-hand panel (see Figure 5). Other problems have regarded the modalities of navigation and movement in the virtual space:

- all the users have experienced initial difficulties in using the keyboard arrows, since they are activated just after having clicked on the virtual stage (a user has never been able to use them);

- all users said they preferred rotator widget (at the top-left corner) than the guiding arrows present on the floor (see Figure 5). However all of them have started using the rotator just after the tester's suggestions, since they were not aware of it. They also said they preferred the keyboard arrows, but sometime they have difficulties in activating them;

- most users go back to the home page by means of browser's back button, since a proper Home button does not exist;

- three users got outside the museums;

Other criticisms and issues raised by users when thinking aloud and during the interview concerned:

- the possibility of directly clicking on a chosen artwork: two users said they would prefer to click directly on the artwork instead of using the icon for getting more information about the artwork. Notice that this icon is sometimes not available either because the user is too close to the artwork or because there is no more information about the artwork, thus generating confusion for the user;

- 3 users have experienced difficulties in finding the search facility at the middle of the top panel;

- 4 users have experienced difficulties in finding the navigate floor plan facility;

- when users got outside the museum they felt guilty and inadequate for the task and they were afraid of having done something wrong. Those three users felt more disorientated during the visit;

- when they did not find the required information, users exploited the textual search to find information;

- the more experienced 3D user (\#2) criticized the quality of artwork during the visit, and more in general the visit experienced. She found the artworks too grainy and in general the navigation not continuous and too jerky (this was the same 
feeling of user \#1). User \#2 would have preferred an reconstructed environment instead of this realistic but not well working one;

- user \#2 would like to enlarge the artwork when she is inside the museum room, instead of doing that in a dedicate web page;

- user \#1 suggested to indicate on the room map where the user is at that moment inside the room. She also suggest to add the map of all the museum floors;

- user \#4 suggested that movements by the arrows should be more bound to prevent user errors

- three users thought that Google Art is very useful for reviewing the artworks of a museum after the visit, while two users found it useful as a pre-visit examination;

- all the users compared the experience with Google Art to Google Street View. User \#2 also noticed the is difficult in the same way;

All the users have really appreciated the zoom facility offered by the Artwork View mode and they spend a lot of time in observing the details of the artworks.

Final results of post hoc questionnaires (see Table 3 ) showed that i) looking for information was very easy; ii) the movement in the hyperspace were quite easy; iii) label's arrows were very comprehensible; iv) information were very exhaustive, and v) the experience was really enjoyable.

\subsubsection{Discussion}

Even if all the users reported some problem in the execution of the tasks, however the global experience was positive. Most of them said they would return to Google Art to spend more time in exploring artworks. After the initial difficulties the navigation and orientation mechanisms become clearer, however most user said the preferred the searching facilities since they are not used to move in a 3D space. The analysis of the results suggested the following re-designs:

- making artworks always clickable, so that user can get anytime more information about them;

- inserting an "Home" button, clear and always present;

- adding the possibility of going back in the visit path;

- making the icon of the right-hand panel more visible and selfexplanatory;

- making the keyboard arrows always active;

- inserting the artwork's name directly on the museum visit;

- adding cross-museum thematic paths by artist;

- enhancing the search tools (adding a cross-museum search by artist, a search restricted to a specific room, etc.);

- adding facilities for the orientation into the virtual space (e.g., "you are here" facility restricted to the orientation in a specific room, a top view of the artworks in a museum floor, the floors map, etc.);

- giving the possibility of re-entering into the museum, if the user is getting outside of.

As already anticipated, in April 2012 Google launched a new version of Google Art project. The new release of Google Art also includes completely new navigation tools and a new interface (for details see ${ }^{6}$ ). Some of the changes matches the user requirements and re-designs emerged from our test (highlighted in bold):

- the default visit is now the "slideshow" view. The user has to voluntary choose the 3D tour, which is now called "Museum view";

- the search tools have been enhanced: users may now browse all the contents by the artists name, the artwork, the type of art, the museum, the country, the city and the collection;

- Google Plus and video hangouts are now integrated on the site, allowing viewers to create "social" galleries;

- the gallery interiors can also be explored directly from within Street View in Google Maps, and it is now impossible to get outside the museum;

- the new tool called "Discover" displays works from different museums around the world and the users can filter artworks by period, artist or type of artwork;

- the learning goals of the project are now especially promoted, thus, as in our Web 3.0 portal, the educational goals are leveraged with respect to other goals. Moreover comments can be added to each painting and the whole collection can then be shared with friends and family. This is particularly ideal tool for students;

- a "you are here" facility has been added on the museum's floor map: when a user is visiting a room a light on the map highlights the current user position;

- when a user is "exploring" an artowork, bread of crumbs show the visit path, and the user can goes back into the visit path

- the artwork's name now is appearing direclty on the museum visit when the user click on the 3D scene with the mouse or when is using the keyboard arrows.

\subsection{Comparison between the two evaluations}

Comparing Table 2 and 3 results show a better trend for Google Art along all the considered dimensions. Considering the user direct observations and their collected opinions, the findings can be compared according to following non-exclusive points of view:

- guidance vs. user initiative. In both the experiments users seem to particularly appreciate guidance for the overview of an environment (e.g. the top view of the all exhibition spaces in the Web 3.0 portal or the overview of a room in Google Art). While they instead prefer to freely move (by means of keyboard arrow) when they visit a room in detail;

- web-based needs of the users. In both the tests users would always be able to click on the artworks to get more information. They would have an home button, and a in Google Art also the possibility to going back on the visit path. All these user's needs derive somehow from the user's habits on the web and emphasize their need for freedom and initiative;

- needs for orientation in the 3D space. In both the experiments users would like to have some kind of "you are here" functionality, such as map of the virtual space highlighting the current user position and orientation, a top view of the museum floor, a way to coming back to start of the visit, a way to going back on the visit path, etc;

\footnotetext{
${ }^{6}$ https://sites.google.com/a/pressatgoogle.com/art-project/press-release
} 
- "back to the reality" needs. The already mentioned request of having a "you are here" facility is also inherited from the experience the user have during the real museum visit. Other emerged requirements such as reading the name of the artwork on the virtual visit, obtaining the more information on an artwork in the same virtual space and not in another browser window, highlight the user's need of having the overall visit experience into the same context, as it happens in the real museum visit experience.

\section{Related work}

Concerning the use of 3D into web-based learning platform [Cerbo et al. 2010] presented a 3D learning environment (called DIEL) where users can interact via avatars. Users can exploit the proximity relationships with other avatars and resources to become immersed in a social constructivist. [Rodriguez-Echavarria et al. 2009] funded semantics and 3D for web-based presentations for public sculptures and monuments in United Kingdom. This work tackles the integration of semantic information to 3D models in web browsers in the domain of cultural heritage where ing the documentation and presentation of items can actually benefit from the association of semantic information to 3D models. [Calori et al. 2008] presented a framework for 3D realtime applications in web browsers that is based on FOSS technologies. In the reported version, a crowd of Roman characters is introduced in the Forum, a highly detailed set of buildings that belong to the Rome Reborn model. The Non Playing Characters (NPCs) "are wandering in the 3D environment between predefined points of interest, while the player is able to move freely". The procedure by which users interacts with the characters (NPCs) is similar to the procedure by which users interact with objects (Art installations) in 150 Digit (a click on a point of interest followed by a camera movement): "When clicked on by the player, a NPC changes its current target to the position of the player, and hence starts walking towards the player. When the NPC is close enough to enter the dialogue level, a series of actions is triggered by the engine: An animation is triggered to change the camera from a wide angle to a close-up perspective".

[Cabral et al. 2007] in a work that use virtual reality technologies to reconstruct and further explore ancient and historic city buildings tried two different approaches in their visualization tool: A joy-stick that simulates a well-known game like interface and a 3D mouse (tracked by a Flock of Birds device). The 3D mouse interface worked with a simple point-and-go simulation: the user would use the 3D mouse to point where to go (controlling the viewing direction of the camera) and the click the left button to go forward and the right button to go backwards. Subjective analyses of users using the joystick interface provided good results on learnability and efficiency. The authors believe this is due to the current knowledge people have using video games. However, efficiency greatly varies among users, most of the times favoring those who are usual videogame players, as our experimental results also demonstrate. On the other hand, the level of satisfaction people showed when using a 3D mouse to navigate was impressive. Almost every user found the 3D mouse interface easy to use and also pleasuring to utilize.

Concerning other application domain, more in general 3D Virtual Environments can help people in learning through direct experience by visualizing concepts and performing tasks in a reproduction of the real world or in completely fictional worlds that are suited to the learning task [Chittaro and Ranon 2007]. For instance, Chittaro and Ranon [Chittaro and Ranon 2007] proposed a case study in which their system was used to build an adaptive 3D Web application for learning how to build interactive 3D graphics content using the eXtensible 3D (X3D) language. Educational Virtual Environments have also the great potential to circumvent physical, safety, and cost constraints during learning and training purposes. Ieronutti and Chittaro [Ieronutti and Chittaro 2007] presented a general architecture that allows content creators to easily integrate virtual humans into Web3D Educational Virtual Environments. In the fields of Intelligent Tutoring Systems and Artificial Intelligence in Education Graesser et al. [Graesser et al. 2005] have proposed a dialogue-based tutoring augmented with 3D interactive simulations.

\section{Conclusion}

In this paper we have described the design and evaluation of a 3D tour of a Web 3.0 portal presenting the exhibitions set up for the celebration of the 150 years of Italian unity. After having described the $3 \mathrm{D}$ tour and the technologies supporting it, we have illustrated the evaluation we conducted of the 3D tour and of a commercial project, Google Art Project, and then we have discussed the results.

The evaluated applications trade off user initiative and guidance by letting the user to freely navigate and by anchoring the $3 \mathrm{D}$ contents in the information structure of the web site and in some sort of proposed navigation. Results have shown that users appraciate both freedom and guidance, even if in different tasks. However these two "opposite" features need to be properly designed (e.g., user wants to be free in selecting all the clickable items, but she need physical boundaries that prevent her errors; guiding arrows must put the user in front of a painting and not in front of a wall, etc.) in order to satisfy final users.

\section{Acknowledgements}

The authors would like to thank: Acknowledgments omitted for blind review.

\section{References}

BRUSILOVSKY, P. 2001. Adaptive hypermedia. User Model. UserAdapt. Interact. 11, 1-2, 87-110.

Burigat, S., AND ChitTARo, L. 2007. Navigation in 3d virtual environments: Effects of user experience and location-pointing navigation aids. International Journal of Human-Computer Studies 65, 11, 945-958.

Cabral, M., Zuffo, M. K., Ghirotti, S., Belloc, O., Nomura, L., NAgamura, M., ANDrade, F., FARIA, R., AND FERRAZ, L. 2007. An experience using x3d for virtual cultural heritage. In Web3D, ACM, O. Gervasi and D. P. Brutzman, Eds., $161-164$.

Calori, L., Camporesi, C., Negri, A., and Pescarin, S. 2008. Virtual rome. In SIGGRAPH Posters, ACM, 101.

Cerbo, F. D., Dodero, G., AND PAPAleo, L. 2010. Integrating a web3d interface into an e-learning platform. In Web3D, ACM, D. G. Aliaga, M. M. Oliveira, A. Varshney, and C. Wyman, Eds., 83-92.

Chittaro, L., And Ranon, R. 2007. Adaptive hypermedia techniques for $3 \mathrm{~d}$ educational virtual environments. IEEE Intelligent Systems 22, 4, 31-37.

Damiano, R., Gena, C., Lombardo, V., Nunnari, F., SupPINI, A., AND CREvolA, A. 2011. 150 digit. Integrating 3d visit and social functions into a web 3.0 learning-oriented approach. In $B W C C A, 136-143$. 
Graesser, A., Chipman, P., Haynes, B. C., And Olney, A. 2005. AutoTutor: an intelligent tutoring system with mixedinitiative dialogue. Education, IEEE Transactions on 48, 4 (Nov.), 612-618.

IERONUtTi, L., AND ChitTARo, L. 2007. Employing virtual humans for education and training in $\mathrm{x} 3 \mathrm{~d} / \mathrm{vrml}$ worlds. Computers \& Education 49, 1, 93-109.

JANKowski, J. 2011. A taskonomy of $3 \mathrm{~d}$ web use. In Web3D, ACM, J. Royan, M. Preda, T. Boubekeur, and N. F. Polys, Eds., 93-100.

Rodriguez-Echavarria, K., Morris, D. C., AND Arnold, D. B. 2009. Web based presentation of semantically tagged $3 \mathrm{~d}$ content for public sculptures and monuments in the uk. In Web3D, ACM, S. N. Spencer, D. W. Fellner, J. Behr, and K. Walczak, Eds., 119-126. 